\title{
nivel de conciencia fonológica en estudiantes con funcionamiento intelectual limítrofe
}

\author{
Level of Phonological fwareness in Students with Borderline Intellectual \\ functioning \\ nível de consciência fonológica em estudantes com funcionamento intelectual \\ limítrofe
}

\author{
María Francisca Muñoz-Oyarce \\ Manuel Monzalve-Macaya \\ Universidad Católica del Maule \\ Alejandro Almonacid-Fierro* \\ Universidad Católica del Maule \\ Universidad Autónoma de Chile \\ Eugenio Merellano-Navarro \\ Universidad Autónoma de Chile
}

Doi: https://doi.org/10.12804/revistas.urosario.edu.co/apl/a.7704

\section{Resumen}

La conciencia fonológica aparece en la literatura como uno de los mayores predictores del aprendizaje $\mathrm{y}$, consecuentemente, como uno de los mecanismos fundamentales para alcanzar el dominio de la lectura. Aunque existe un amplio cuerpo de investigación en este ámbito, hay escasos estudios que muestren cómo estas habilidades fonológicas se desarrollan en estudiantes con características intelectuales o cognitivas atípicas. El presente estudio tiene por objetivo caracterizar el nivel de habilidades de conciencia fonológica en estudiantes con funcionamiento intelectual limítrofe atendidos en Programas de Integración Escolar, a través de la aplicación de la Prueba de Evaluación de Conciencia Fonológica. Los participantes fueron 37 estudiantes con diagnóstico de función intelectual limítrofe, que cursaban entre primer y tercer año de educación primaria, con diagnóstico de función intelectual limítrofe. Los

* Dirigir correspondencia a: Alejandro Almonacid-Fierro, Universidad Católica del Maule. Dirección: Av. San Miguel 3605, Talca, Región del Maule, Chile. Correo electrónico: aalmonacid@ucm.cl

ORCID ID: http://orcid.org/0000-0002-8328-017X

Para citar este artigo: Muñoz-Oyarce, M. F., Monzalve-Macaya, M., Almonacid-Fierro, A., \& Merellano-Navarro, E. (2020). Nivel de conciencia fonológica en estudiantes con funcionamiento intelectual limítrofe. Avances en Psicología Latinoamericana, $38(2), 1-14$. https://doi.org/10.12804/revistas.urosario.edu.co/apl/a.7704 
resultados muestran un descenso heterogéneo importante en el desarrollo de las habilidades fonológicas evaluadas, lo que preliminarmente indicaría un alto grado de riesgo en la adquisición de nuevos aprendizajes, así como también en la proyección y avance académico de los estudiantes. Adicionalmente, se discuten las implicancias para la práctica pedagógica, política y futuras investigaciones en este ámbito.

Palabras claves: conciencia fonológica; aprendizaje; escolarización.

\section{fbstract}

$\overline{\text { Current literature shows phonological awareness is one }}$ of the best reading learning predictors and, consequently, one of the core skills to develop reading. Although there is a large body of research in this area, there are few studies that show how these phonological skills develop in students with atypical intellectual or cognitive characteristics. The objective of this study is to characterize the level of phonological awareness skill in students with borderline intellectual functioning attending School Integration Programs. To assess the level of phonological awareness, the Phonological Awareness Test was used. The participants were 37 students in the first to third years of primary education with a diagnosis of borderline intellectual functioning. The results showed an important heterogeneous decrease in the development of the phonological skills assessed, which indicates a high degree of risk in the acquisition of reading, as well as in the projection and academic progress of these students. The implications for practice, policies, and future research in this area are discussed.

Keywords: Phonological awareness learning; schooling.

\section{Resumo}

$\overline{\text { A consciência fonológica aparece dentro da literatura }}$ como um dos maiores preditores da aprendizagem e consequentemente, como um dos mecanismos fundamentais para alcançar o domínio da leitura. Ainda que existe um amplo corpo de pesquisa neste âmbito, existem escassos estudos que mostram como estas habilidades fonológicas se desenvolvem em estudantes com características intelectuais e/ou cognitivas atípicas. O presente estudo tem por objetivo caracterizar o nível de habilidade de Consciência Fonológica em estudantes com Funcionamento Intelectual Limítrofe atendidos em Programas de Integração Escolar, através da aplicação da Prova de Avaliação de Consciência Fonológica. Os participantes foram 37 estudantes que estavam entre primeiro e terceiro ano de ensino básico com diagnóstico de Função Intelectual Limítrofe. Os resultados mostram um descenso heterogêneo importante no desenvolvimento das habilidades fonológicas avaliadas, o que preliminarmente indicaria um alto grau de risco na aquisição de novas aprendizagens assim como também na projeção e avanço acadêmico dos estudantes. As implicâncias para a prática pedagógica, política e futuras pesquisas neste âmbito são discutidas.

Palavras-chave: consciência fonológica; aprendizagem; escolarização.

Uno de los propósitos fundamentales de la reforma educacional iniciada en Chile durante el presente gobierno es disminuir las brechas sociales y asegurar la participación en el país de los niños, jóvenes y adultos como personas críticas, creativas y reflexivas, para de esta forma promover el desarrollo de un país integrado a la cultura y la sociedad del siglo XXI. En este contexto, el Ministerio de Educación ha propuesto el Plan Nacional de la Lectura 2015-2020 (MINEDUC, 2015) con la idea de lograr una sociedad que disfrute de la lectura y la vea como una posibilidad de aumentar el capital cultural y consecuentemente aspirar a un mejor desarrollo humano. En este sentido, la política pública considera la educación un derecho social que promueve un aprendizaje integral que abre sus puertas hacia una concepción inclusiva e intercultural capaz de acoger, sin distinción alguna, a todos los estudiantes —niños y niñas, jóvenes y adultos — que asisten diariamente a las aulas en todo el territorio chileno (Darling-Hammond, 2012). 
De acuerdo con los trabajos de Medina y Gajardo (2010), el nivel de alfabetización reportado por la población chilena es positivo, cercano al $97 \%$. En este sentido, podemos afirmar que la sociedad actual exige a los individuos contar con mayores competencias en lectura, lo cual demanda de los miembros de la sociedad chilena que sean personas que no solo sean capaces de decodificar el significado de las palabras, sino que también se espera que puedan manejar información de distinto tipo, vale decir, leer comprensivamente los textos, comunicarse de forma eficaz, resolver problemas, desarrollar un pensamiento crítico, interpretar y evaluar los mensajes de los medios de comunicación, en definitiva que sean capaces de responder a un entorno en constante cambio.

La investigación en el ámbito de la lectura y las dificultades encontradas en su aprendizaje han conducido a la identificación de competencias consideradas facilitadoras de la alfabetización que deberían ser abordadas desde la educación infantil (Porta \& Ison, 2011). Entre las más destacadas por la literatura están el lenguaje oral, el conocimiento del material impreso y las habilidades de conciencia fonológica - las habilidades para reflejar los sonidos de la propia lengua oral- (Cruz et al., 2014; Melby-Lervåg, Lyster \& Hulme, 2012; Reis, Proenca \& Alves-Martins, 2015). Esta última se define como "la habilidad para segmentar y manipular las palabras en operaciones de identificación de sílabas, fonemas, sonidos iniciales y finales de una palabra, combinación de fonemas para formar nuevas palabras y detección de rimas" de acuerdo con lo señalado por Flores, Castro y Arias (2011, p. 241).

Las habilidades fonológicas han sido objeto de estudio y de diversas investigaciones, nacionales e internacionales, dada su influencia significativa en el aprendizaje de la adquisición del proceso lector. Anthony y Francis, citados en Varela y de Barbierie (2015), plantean que desde el punto de vista evolutivo la conciencia fonológica se desarrolla fuertemente durante el periodo comprendido entre las edades de los 4 y 8 años. En primer lugar, se desarrolla la conciencia silábica y posteriormente se adquiere el manejo de habilidades fonémicas una vez que los niños aprenden a leer y escribir (p. 20).

Es indudable que el desarrollo social, cognitivo, económico y cultural que suscita el aprendizaje de la lectura abre un mundo de posibilidades dentro de todo el ciclo vital de un ser humano. Sin embargo, un porcentaje no menor de las personas queda excluido de este derecho por causas multifactoriales, como es el caso de las personas diagnosticadas con función intelectual limítrofe (FIL), los cuales, debido a su patología, no logran alcanzar los niveles mínimos de decodificación del lenguaje escrito (Lazcano-Ponce et al., 2013).

La escritura como la lectura necesitan de un oído fonemático preciso - aquel mecanismo cerebral que soporta el desarrollo de habilidades fonológicas - , que asegure la identificación de las unidades léxicas de la lengua. Lo anterior, con la idea de permitir comparar la forma ortográfica de la palabra escrita - secuencia de letras en la organización del engrama - con las representaciones mentales de palabras (Feld, 2014).

En este sentido, determinar el nivel de habilidades fonológicas desarrolladas en los niños y niñas con FIL en los primeros años de escolaridad sería el punto de partida para una reflexión profesional respecto a la comprensión de sus dificultades en el aprendizaje lector como proceso facilitador en la articulación de todos los aprendizajes, tanto a nivel instrumental como adaptativo (Aravena, 2014). Por tanto, el presente estudio se inscribe en la concepción que la inteligencia limítrofe no sería un trastorno mental, sino más bien una forma de funcionamiento intelectual, que podría presentar diferentes dificultades en el aprendizaje, las cuales no constituirían un trastorno por sí mismas, sino que serían características constitutivas de la FIL (Alvarán, Sánchez \& Restrepo-Ochoa, 2016).

En relación con la FIL, se puede conceptualizar como la frontera que delimita el funcionamiento intelectual normal y las dificultades intelectuales 
y de desarrollo, cuestión que ha sido propuesta por la American Association on Intellectual and Developmental Disabilities con objeto de sustituir el termino deficiencia (Salvador-Carulla, et al., 2013; Santos \& Morato, 2012). En términos más específicos, corresponde a niños y niñas que presentan un QI (coeficiente intelectual) de 70 a 84, medido con la escala de inteligencia de Wechsler (Água-Dias, 2016).

Las causas de una inteligencia descendida son variadas con factores de riesgo tales como prematuridad, factores genéticos, ambiente familiar con baja alfabetización parental, cuidados prenatales deficientes o inexistentes, exposición prenatal al alcohol o drogas. Por otro lado, de acuerdo con investigaciones recientes, existen alteraciones relacionadas con la FIL como la dislexia, la discalculia, la perturbación del aprendizaje no verbal, el síndrome fetal alcohólico, el síndrome velocardiofacial, el síndrome de Williams y las perturbaciones del espectro autista, entre otros (Cooter \& Cooter, 2004; Salvador-Carulla et al., 2013).

De acuerdo con una revisión sistemática de la literatura con personas con FIL (Karande, Kanchan \& Kulkarni, 2008), se enfatizan factores relevantes respecto de la prevención en la adquisición de diversos aprendizajes, entre los que se destaca la lectura. Los resultados indican que las habilidades de lectura de los adultos son deficientes: el 17\% se definió como semiletrado y el $12 \%$ como no lector. Con respecto a otras destrezas estudiadas relacionadas con la lectura y la ortografía, el desempeño en la mayoría de las otras tareas fue también deficiente — procesamiento auditivo, habilidades fonológicas y sintácticas y comprensión de lectura- (Kortteinen, citado en Peltopuro et al. 2014). En este sentido y siguiendo los hallazgos de diversos investigadores, potenciar el desarrollo de habilidades fonológicas sería el inicio de la activación cerebral y pedagógica hacia la adquisición del aprendizaje de la lectura como un medio de articulación social y cultural indispensable para mejorar la calidad de vida de los niños y niñas con diagnóstico de FIL
(Bravo, 2016; Porta, 2012; Valdivia, Medina \& San Martín, 2014). De acuerdo con Feld (2014), "la adquisición del proceso lector se puede interpretar de tres maneras: a) el conocimiento fonológico es un requisito para el aprendizaje de la lectura, $b$ ) es un producto del aprendizaje de la lectura y c) ambos están relacionados bidireccionalmente" (p. 73). Ahora bien, independientemente de la interpretación, la intervención de ellas facilitaría la adquisición y metacognición del conocimiento del lenguaje como un código escrito, vale decir, lo que algunos autores conceptualizan como conocimiento metalingüístico, como una subcategoría de la metacognición.

En este contexto, desde temprana edad, aproximadamente de los tres años en adelante, los niños y niñas estructuran los cimientos cognitivos necesarios para la asimilación del lenguaje escrito, cuestión que se da preferentemente por medio del juego simbólico, en que los niños comprenden narraciones de diferentes niveles de complejidad. Lo anterior, les permitiría desarrollar el aprendizaje bajo un simbolismo directo relacionado posteriormente con la adquisición de la lectoescritura. A partir de este proceso, el niño sería capaz de llegar a comprender la convencionalidad del lenguaje escrito, por medio de la percepción paulatina de que los símbolos son representaciones arbitrarias. No obstante, estas son efectivas para la comunicación, pues permiten tomar conciencia del lenguaje como un código y de que este es un código arbitrario y convencional, lo que según van Kleeck (1994) correspondería al desarrollo psicolingüístico.

Para algunos autores, el progreso del desarrollo psicolingüístico, así como el nivel sociocultural al que pertenecen los niños, podría también ser un factor determinante en el desarrollo de habilidades fonológicas y cognitivas superiores que le permitan adquirir el reconocimiento visual de las palabras escritas, asociando los signos gráficos a su significado. Por ello sería relevante una intervención temprana, sistemática y pertinente a las necesidades que presentan los niños de manera individual (Varela \& De Barbieri, 2015). Desde 
otra perspectiva, el trabajo de Flórez-Romero et al. (2005) establece que, a medida que avanza la escolarización, aumentan los niveles de metacognición, expresados en lectura, comprensión lectora, metacognición en escritura y producción escrita, cuestión que referiría fundamentalmente al aumento de los grados escolares, vale decir, la edad de los niños.

El objetivo del presente estudio es caracterizar el nivel de habilidades de conciencia fonológica - considerando implícitamente el desarrollo de la conciencia silábica y la conciencia fonémicaen estudiantes con FIL atendidos en Programas de Integración Escolar (PIE), para describir el nivel de conciencia silábica y fonémica alcanzado en niños y niñas entre los 6 y 8 años, pertenecientes a establecimientos educacionales de la ciudad de Talca, Chile.

\section{Metodología}

El diseño de investigación representado en este estudio es no experimental, de tipo transeccional descriptivo exploratorio, con el cual no se manipulan las variables independientes y solo se observan lo fenómenos para posteriormente explicarlos (Briones, 2002; Hernández, Fernández \& Baptista, 2010).

Se identificó a todos los niños que ingresaron a la plataforma PIE en 2016 de la ciudad de Talca. Esta plataforma se utilizó como recurso para identificar a los colegios y estudiantes con el fin de tomar una muestra, es decir, un subgrupo de la población de interés de la que se recolectaron datos (Hernández, Fernández \& Baptista, 2010). El paso siguiente fue contactar a los directores y profesores de cada establecimiento, con el fin de presentarles el proyecto y enviar el consentimiento informado con los niños. La muestra final estuvo conformada por todos los niños autorizados por sus padres que presentaron el consentimiento informado firmado.
La $n$ final fue de 37 escolares distribuidos en diferentes establecimientos de la ciudad de Talca. Los niños y niñas que participaron fueron evaluados entre los meses de abril y junio de 2017, a cargo de profesionales del área de psicopedagogía y educación diferencial, que fueron capacitados en la aplicación y corrección del instrumento específico.

Los datos fueron obtenidos por medio de una prueba estandarizada, que dio como resultado una muestra no probabilística por conveniencia, debido a que las características de los participantes están particularmente delimitadas por el investigador.

La muestra fue orientada según los siguientes criterios de inclusión:

- Los niños presentan diagnóstico de FIL.

- Tienen edades entre los 6 y 8 años.

- Pertenecen al PIE de la ciudad de Talca.

Tabla 1.

Caracterización de la muestra

Edad (años)

$\mathrm{X} \quad \mathrm{DE}$

Sexo

$\begin{array}{ll}7.4 & 0.7\end{array}$

Mujeres ( $\mathrm{n}=14)$

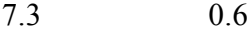

Total $(\mathrm{n}=37)$

\begin{tabular}{l}
$7.3 \quad 0.6$ \\
\hline
\end{tabular}

Curso

fi

$\%$

$1^{\circ}$ Básico

13

35.1

$2^{\circ}$ Básico

21

56.8

$3^{\circ}$ Básico

3

8.1

Para cumplir con el objetivo propuesto, se optó por utilizar la Prueba de Evaluación de Conciencia Fonológica (PECFO) elaborada en 2013 (Valera \& De Barbieri, 2015). Este instrumento permite evaluar la habilidad metafonológica relacionada con la sílaba y el fonema en los niños de 4 y 7 años y 11 meses, 
que consta de 11 subpruebas divididas en dos secciones: una sección de conciencia silábica, la cual contiene seis subpruebas que evalúan segmentación silábica, identificación de sílaba inicial y final, omisión de sílaba inicial y final e inversión silábica, y otra de conciencia fonémica la cual contiene cinco subpruebas que evalúan la identificación de fonema inicial y final, omisión de fonema inicial y síntesis fonémica. Una vez aplicada, se logró establecer el nivel de conciencia fonológica alcanzado por el niño/a con relación a su edad cronológica y el inicio de su aprendizaje lector. El nivel de rendimiento se estableció a partir de los valores normativos presentados por Valera y de Barbieri (2015).

El mencionado instrumento cuenta con un manual y un set de láminas, así como de un protocolo de registro general y una hoja de registro especial para la prueba 1. Está conformado por diez subpruebas divididas en dos secciones: conciencia silábica, con seis ítems y cuatro subpruebas de conciencia fonológica. A su vez, cada una de las secciones presenta tareas específicas (tabla 2).

\section{Resultados}

La tabla 1 presenta la caracterización de la muestra estudiada, en la que fueron evaluados un total de 37 estudiantes (23 hombres y 14 mujeres) de educación básica, diagnosticados con FIL, pertenecientes a los PIE de la ciudad de Talca.

La tabla 3 muestra los valores medios y la desviación estándar (DE) de los ítems correspondientes a conciencia silábica y conciencia fonémica de hombres y mujeres con relación a la edad. Los resultados muestran que en la dimensión conciencia silábica, todos los ítems aumentan en función de la edad, a excepción de la identificación silábica final y omisión de sílaba inicial. En la dimensión conciencia fonémica, el único ítem que no posee un aumento en función de la edad es la omisión fonema final, que evidencia una leve disminución a $\operatorname{los} 8$ años a diferencia de $\operatorname{los} 7$ años.

La tabla 4 muestra los valores medios y la DE de los 10 ítems y del total que conforman ambas dimensiones: conciencia silábica y conciencia fonémica, en relación con la edad en los hombres, donde se observa que en el caso de la conciencia silábica, esta va aumentando con el transcurso de la edad; de la misma forma ocurre a nivel de conciencia fonémica.

A los 8 años, específicamente, reflejaron un mejor rendimiento en la habilidad de omisión de sílaba inicial; no obstante, el más descendido se encuentra a la edad de 6 años en la identificación de sílaba final.

Tabla 2.

Secciones Prueba de Evaluación de Conciencia Fonológica

\begin{tabular}{ccc}
\hline & Sección I & Sección II \\
Conciencia Silábica & Conciencia Fonémica \\
\hline I. & Segmentación silábica & Identificación fonema inicial \\
II. & Identificación sílaba inicial & Identificación fonema final \\
III. & Identificación sílaba final & Omisión fonema inicial \\
IV. & Omisión sílaba inicial & Síntesis fonémica \\
V. & Omisión sílaba final & - \\
IV. & Inversión silábica & -
\end{tabular}

Fuente: elaborado a partir de PECFO, 2013. 
Tabla 3.

Valores medios y DE de los ítems correspondientes a conciencia silábica y conciencia fonémica de hombres y mujeres con relación a la edad cronológica, según resultados obtenidos de la aplicación de PECFO

\begin{tabular}{|c|c|c|c|c|c|c|c|c|}
\hline \multirow{2}{*}{ Edad } & \multicolumn{2}{|c|}{6 años } & \multicolumn{2}{|c|}{7 años } & \multicolumn{2}{|c|}{8 años } & \multicolumn{2}{|c|}{ Todos } \\
\hline & $\mathrm{X}$ & $\mathrm{DE}$ & $\mathrm{X}$ & DE & $\mathrm{X}$ & $\mathrm{DE}$ & $\mathrm{X}$ & $\mathrm{DE}$ \\
\hline $\mathrm{n}$ & \multicolumn{2}{|c|}{5} & \multicolumn{2}{|c|}{22} & \multicolumn{2}{|c|}{10} & \multicolumn{2}{|c|}{37} \\
\hline \multicolumn{9}{|l|}{ Conciencia Silábica } \\
\hline Seg. silábica & 3.2 & 1.3 & 3.9 & 0.9 & 4.6 & 0.5 & 4 & 1.0 \\
\hline Iden. sílaba inicial & 3.8 & 1.1 & 4.1 & 1.08 & 4.2 & 0.8 & 4.1 & 0.9 \\
\hline Iden. sílaba final & 2.8 & 0.8 & 3.3 & 1.7 & 3.2 & 1.4 & 3.2 & 1.5 \\
\hline Omisión sílaba inicial & 3.4 & 1.3 & 3.3 & 1.8 & 4.1 & 1.2 & 3.5 & 1.6 \\
\hline Omisión sílaba final & 2.4 & 1.1 & 3.4 & 1.73 & 4.2 & 1.3 & 3.5 & 1.6 \\
\hline Inversión silábica & 2.0 & 1.2 & 2.2 & 1.53 & 3.1 & 1.4 & 2.4 & 1.49 \\
\hline Conciencia silábica total & 19.33 & 2.0 & 17.82 & 4.2 & 22.6 & 4.6 & 19.4 & 4.6 \\
\hline \multicolumn{9}{|l|}{ Conciencia fonémica } \\
\hline Iden. fonema inicial & 3 & 1.5 & 3.7 & 1.2 & 4.3 & 1.3 & 3.8 & 1.3 \\
\hline Iden. fonema final & 2 & 2 & 2.3 & 1.8 & 2.9 & 1.3 & 2.4 & 1.7 \\
\hline Omisión fonema inicial & 1.6 & 1.5 & 2.3 & 1.4 & 3.1 & 1.3 & 2.6 & 1.4 \\
\hline Omisión fonema final & 2.8 & 1.3 & 3.4 & 1.36 & 3.3 & 1.8 & 3.3 & 1.4 \\
\hline Conciencia fonémica total & 8.7 & 2.3 & 10.8 & 7.9 & 12.3 & 4.4 & 10.9 & 8.0 \\
\hline
\end{tabular}

Tabla 4.

Valores medios y DE de los items correspondientes a conciencia silábica y conciencia fonémica de los hombres con relación a la edad cronológica, según resultados obtenidos de la aplicación de PECFO

\begin{tabular}{|c|c|c|c|c|c|c|c|c|}
\hline \multirow{2}{*}{ Edad } & \multicolumn{2}{|c|}{6 años } & \multicolumn{2}{|c|}{7 años } & \multicolumn{2}{|c|}{8 años } & \multicolumn{2}{|c|}{ Todos } \\
\hline & $\mathrm{X}$ & $\mathrm{DE}$ & $\mathrm{X}$ & $\mathrm{DE}$ & $\mathrm{X}$ & $\mathrm{DE}$ & $\mathrm{X}$ & $\mathrm{DE}$ \\
\hline $\mathrm{N}$ & 3 & & 14 & & 6 & & 23 & \\
\hline \multicolumn{9}{|l|}{ Conciencia silábica } \\
\hline Seg. silábica & 3.3 & 1 & 4.2 & 0.9 & 4 & 1.1 & 4 & 1 \\
\hline Iden. sílaba inicial & 3.3 & 1.3 & 4.1 & 1 & 4 & 1.3 & 3.9 & 1.1 \\
\hline Iden. sílaba final & 2.5 & 0.6 & 3.5 & 1.9 & 3.3 & 1.4 & 3.3 & 1.6 \\
\hline Omisión sílaba inicial & 2.5 & 1 & 3.4 & 1.9 & 4.3 & 1.2 & 3.5 & 1.6 \\
\hline Omisión sílaba final & 2 & 1.4 & 3.6 & 1.9 & 4.2 & 1.3 & 3.5 & 1.8 \\
\hline Inversión silábica & 2.5 & 0.6 & 2.1 & 1.8 & 3.2 & 1.5 & 2.4 & 1.6 \\
\hline Conciencia silábica total & 16 & 3.7 & 20.8 & 6.1 & 23 & 5.8 & 20.6 & 5.9 \\
\hline \multicolumn{9}{|l|}{ Conciencia fonémica } \\
\hline Iden. Fonema inicial & 2.5 & 1.9 & 3.9 & 1.2 & 4 & 1.5 & 3.7 & 1.5 \\
\hline Iden. Fonema final & 1.5 & 2.4 & 2.5 & 2 & 3.2 & 1.6 & 2.5 & 2 \\
\hline Omisión fonema inicial & 2 & 1.4 & 2.8 & 1.5 & 3.2 & 1.3 & 2.7 & 1.4 \\
\hline Omisión fonema final & 2.5 & 1.7 & 4 & 0.8 & 3 & 2.1 & 3.5 & 1.5 \\
\hline Conciencia fonémica total & 8.5 & 6.5 & 13.2 & 3.9 & 13.3 & 5.9 & 12.4 & 5 \\
\hline
\end{tabular}


A nivel de conciencia fonémica igualmente logran un mejor desarrollo a los 8 años a nivel de identificación de fonema inicial; a los 6 años reflejan la habilidad más descendida en el ítem de identificación de fonema final.

En la tabla 5 se muestran los valores medios y DE de los 10 ítems y del total que conforman ambas dimensiones de conciencia silábica y conciencia fonológica en las mujeres, donde se observa un aumento de los 6 a los 7 años y luego una disminución a los 8 años. El mejor rendimiento en habilidades de conciencia silábica lo obtuvieron a los 7 años, la identificación de sílaba inicial fue su mayor fortaleza. Del mismo grupo, la debilidad se observa en la edad de 8 años, la inversión silábica fue su mayor debilidad.

La tabla 6 muestra el desempeño de la conciencia fonémica a los 7 años, la identificación de fonema inicial fue la mayor fortaleza. No obstante, su debilidad se observa a nivel de los 6 años, el ítem más descendido fue la síntesis fonémica.

\section{Discusión}

La FIL afecta a la población infantil como una metacondición caracterizada por dificultades cognitivas de desarrollo social y adaptación al contexto. El bajo rendimiento académico es causa frecuente de deserción escolar y con ello el desarrollo potencial de una amplia gama de problemáticas mentales al llegar a la adolescencia (Lazcano-Ponce et al., 2013). Considerando que los estudiantes con FIL tienen dificultades para cumplir con los requisitos académicos, es importante considerar la intervención temprana para evitar que estas impidan el

Tabla 5.

Valores medios y DE de los items correspondientes a conciencia silábica y conciencia fonémica de las mujeres con relación a la edad cronológica, según resultados obtenidos de la aplicación de PECFO

\begin{tabular}{|c|c|c|c|c|c|c|c|c|}
\hline \multirow{2}{*}{ Edad } & \multicolumn{2}{|c|}{6 años } & \multicolumn{2}{|c|}{7 años } & \multicolumn{2}{|c|}{8 años } & \multicolumn{2}{|c|}{ Todos } \\
\hline & $\mathrm{X}$ & $\mathrm{DE}$ & $\mathrm{X}$ & $\mathrm{DE}$ & $\mathrm{X}$ & $\mathrm{DE}$ & $\mathrm{X}$ & $\mathrm{DE}$ \\
\hline $\mathrm{N}$ & \multicolumn{2}{|c|}{2} & \multicolumn{2}{|c|}{8} & \multicolumn{2}{|c|}{4} & \multicolumn{2}{|c|}{14} \\
\hline \multicolumn{9}{|l|}{ Conciencia silábica } \\
\hline Seg. silábica & 3.5 & 2.1 & 4.2 & 1.1 & 4.3 & 0.6 & 4.1 & 1.1 \\
\hline Iden. sílaba inicial & 4.0 & 1.4 & 4.6 & 0.5 & 4.3 & 1.2 & 4.4 & 0.8 \\
\hline Iden. sílaba final & 3.0 & 1.4 & 3.4 & 1.5 & 2.0 & 2 & 3.1 & 1.6 \\
\hline Omisión sílaba inicial & 4.5 & 0.7 & 3.3 & 1.9 & 3.7 & 0.6 & 3.6 & 1.6 \\
\hline Omisión sílaba final & 2.5 & 0.7 & 4.2 & 1.1 & 2.0 & 1 & 3.5 & 1.4 \\
\hline Inversión silábica & 1.5 & 2.1 & 2.9 & 1.2 & 1.3 & 1.5 & 2.4 & 1.4 \\
\hline Conciencia Silábica total & 19 & 2.8 & 22.7 & 4.8 & 17.7 & 5 & 21.1 & 4.9 \\
\hline \multicolumn{9}{|l|}{ Conciencia fonémica } \\
\hline Iden. Fonema inicial & 3.0 & 1.4 & 4.1 & 0.8 & 3.7 & 1.5 & 3.9 & 1.0 \\
\hline Iden. Fonema final & 2.0 & 1.4 & 2.6 & 1.4 & 1.7 & 1.5 & 2.3 & 1.4 \\
\hline Omisión fonema inicial & 0.5 & 0.7 & 2.9 & 1.2 & 2.0 & 2.0 & 2.4 & 1.5 \\
\hline Omisión fonema final & 2.5 & 0.7 & 3.4 & 1.3 & 1.7 & 1.5 & 2.9 & 1.4 \\
\hline Conciencia fonémica total & 8.0 & 2.8 & 13.0 & 2.7 & 9.0 & 6.2 & 11.4 & 4.0 \\
\hline
\end{tabular}


Tabla 6.

Valores medios y Desviación Estándar del puntaje total PECFO correspondiente a conciencia silábica y conciencia fonémica de los hombres y mujeres con relación a la edad cronológica

\begin{tabular}{|c|c|c|c|c|c|c|c|c|}
\hline \multirow{2}{*}{ Edad (años) } & \multicolumn{2}{|c|}{6} & \multicolumn{2}{|c|}{7} & \multicolumn{2}{|c|}{8} & \multicolumn{2}{|c|}{ Todos } \\
\hline & $\mathrm{X}$ & $\mathrm{DE}$ & $\mathrm{X}$ & $\mathrm{DE}$ & $\mathrm{X}$ & $\mathrm{DE}$ & $\mathrm{X}$ & $\mathrm{DE}$ \\
\hline \multicolumn{9}{|l|}{ Hombres } \\
\hline Puntaje total PECFO & 24.5 & 9.5 & 34.1 & 9.6 & 36.3 & 10.7 & 33 & 10.3 \\
\hline \multicolumn{9}{|l|}{ Mujeres } \\
\hline Puntaje total PECFO & 27 & 5.7 & 35.7 & 6.9 & 26.7 & 10.2 & 32.5 & 8.2 \\
\hline
\end{tabular}

avance del aprendizaje, como lo demuestran estudios como el de Malik, Rehman y Hanif, (2012). Debido a que estos niños no presentan características físicas ni sociales que determinen tempranamente su condición, es la escuela en donde principalmente se visualizan las primeras barreras sintomáticas. Por ello este estudio se centró en un grupo de 37 niños y niñas entre los 6 y 8 años con diagnóstico de FIL pertenecientes al PIE.

Del total de evaluados, 23 son varones y 14 son mujeres (tabla 1), cuestión que podría coincidir con lo reportado por la literatura respecto a que hay mayor cantidad de estudiantes hombres que presentan FIL. De acuerdo con lo reportado por Artigas-Pallares et al., citados en Alvarán, Sanchez y Restrepo-Ochoa (2016), "la inteligencia limítrofe se presenta con mayor frecuencia en el sexo masculino respecto al sexo femenino" (p. 136). Lo anterior, en el presente estudio, no es posible afirmarlo debido al tamaño de la muestra.

Por otra parte, hombres y mujeres con FIL presentan una alta probabilidad de desarrollar dificultades de aprendizaje aun cuando no existe claridad respecto de la prevalencia en un dominio instrumental específico; su condición cognitiva se establece como una disfunción en habilidades de procesamiento y otros procesos mentales como la memoria de trabajo que de alguna manera determinan el desempeño y la forma de aprender en comparación con estudiantes de su misma edad y con características intelectuales consideradas dentro de los rangos normales (Atuesta, Vásquez \& Urrego, 2008; Baglio et al., 2014; Bermúdez, 2010).

Si bien podrían ser variadas las dificultades de aprendizaje transitorias o permanentes que pudiesen desarrollar los niños con FIL (Bermúdez, 2010), en esta investigación se examinó el desarrollo de habilidades fonológicas subyacentes al aprendizaje de la lectura, considerada como una herramienta transversal de integración escolar, cultural y social, que les permitiría a los estudiantes con FIL alcanzar mejores competencias curriculares y sociales.

El desarrollo de la conciencia fonológica sigue siendo el mayor predictor en el aprendizaje de la lectura. Alcanzar la reflexión consciente de su proceso es el motor de ensamblaje entre los mecanismos de conversión fonema-grafema, caracterizado por la capacidad generativa y de autoaprendizaje del código fonológico. Extraer el significado a partir de dicho proceso es en exclusiva un aprendizaje lector (Hassiotis, 2015).

En las últimas décadas, diversas investigaciones han destacado que el proceso metafonológico, debe ser adquirido por medio de su relación con el alfabeto. Lo anterior, en el entendido que todos los seres humanos son potenciales sujetos lectores. Dicha dependencia estaría condicionada, en algunos casos, a la intervención de la escuela (Alegría, 2006). 
En este sentido, como una forma de planear la enseñanza, las acciones de prevención y posteriores intervenciones, es crucial identificar el nivel de desarrollo de las habilidades fonológicas que alcanzan los estudiantes en el proceso inicial de aprendizaje de la lectura, especialmente aquellos estudiantes que presentan alguna necesidad educativa específica, como es la FIL, dada la complejidad que podría producir en estos grupos de estudiantes.

Aunque la muestra no fue robusta para hacer generalizaciones y diferenciaciones tomando el sexo de los participantes, en el presente estudio se puede observar que los niveles de conciencia silábica y fonémica tanto en hombres como en mujeres no presentan diferencias importantes, aun cuando la conciencia silábica va aumentando con la edad en los varones. No obstante, en las mujeres aumenta al inicio y disminuye a $\operatorname{los} 8$ años.

Respecto de los resultados obtenidos por los hombres en los ítems correspondientes a conciencia silábica — segmentación silábica, identificación de sílaba inicial, identificación de sílaba final, omisión de sílaba final e identificación silábica-, el mejor rendimiento se obtuvo a los 8 años, quienes reflejaron un mejor rendimiento en la habilidad de omisión de sílaba inicial. No obstante, el más descendido se encuentra a los 6 años en la identificación de sílaba final.

En el ítem: conciencia fonémica - identificación fonema inicial, identificación fonema final, omisión de fonema inicial y síntesis fonémica-, los varones presentan mejor rendimiento igualmente a la edad de 8 años. La habilidad más descendida en el ítem mencionado se refleja a los 6 años en la identificación de fonema final.

Del grupo de mujeres evaluadas, su mejor rendimiento en habilidades de conciencia silábica la obtuvieron a los 7 años, la identificación de sílaba inicial fue su mayor fortaleza. Del mismo grupo, la debilidad se observa a la edad de 8 años, la inversión silábica fue su mayor debilidad. El género femenino presenta un mejor desarrollo de conciencia fonémica a los 7 años, la identifi- cación de fonema inicial fue la mayor fortaleza. La debilidad a nivel de conciencia fonémica se presenta a los 6 años, el ítem más descendido fue la síntesis fonémica.

Los resultados obtenidos revelan un rendimiento deficitario en el desarrollo de la conciencia fonológica más en hombres que en mujeres, cuestión que es coincidente con la investigación de Aravena (2014), quien entre sus hallazgos destaca que el $56 \%$ de los sujetos estudiados presenta bajo desarrollo de conciencia fonológica. Por otro lado, el nivel alcanzado por los estudiantes varones muestra que el rendimiento obtenido a los 6 años posee un $50 \%$ de rendimiento deficitario frente a los 7 años, edad en la que el porcentaje aumenta a $61.5 \%$. Solo un $25 \%$ a los 6 años y un $7.7 \%$ a los 7 años se clasifican en la categoría normal. Respecto del grupo femenino, un $44.4 \%$ de las mujeres se presenta en la categoría de un desarrollo normal a los 7 años; sin embargo, el 100\% de ellas se agrupa en la categoría en riesgo a los 6 años, mientras que a los 7 años un $44 \%$ estaría en déficit y un $11.1 \%$, en riesgo.

Los resultados muestran que en ambos grupos ninguno de ellos alcanzó el desarrollo adecuado en más de una tarea evaluada. Al comparar los resultados con investigaciones similares (Melby-Lervåg, Lyster \& Hulme, 2012), podemos señalar que los estudiantes evaluados en esta investigación presentarían dificultades para transformarse en lectores competentes, ya que el nivel de conciencia fonológica alcanzado les dificultaría acceder al significado de los textos escritos, toda vez que se comprende que las habilidades de conciencia fonológica son parte constitutiva, entre otras, de la competencia denominada alfabetización emergente (Santos \& Barrera, 2017). Sin embargo, las definiciones curriculares, las prácticas pedagógicas de los profesores y fundamentalmente la calidad de la escuela serán determinantes para lograr el éxito académico en estos niños (Molina, 2016).

Se destaca el trabajo realizado por Bravo-Valdivieso, Villalón y Orellana (2004), donde se realizó 
seguimiento desde primer año de enseñanza básica hasta tercer año, para el que se determinaron la evolución del proceso lector y las variables iníciales más predictivas del rendimiento final. En dicho estudio, los resultados arrojaron que el "mejor rendimiento al finalizar el tercer año básico fueron aquellos que al iniciar su escolaridad tenían mejor desarrollo de la conciencia fonológica y habilidades verbales para encontrar analogías y ordenar oraciones" (p. 15).

Por otro lado, la investigación en esta área señala que la dificultad en el desarrollo de la conciencia fonológica va aumentando con la edad cronológica y por ende con la articulación de los niveles de escolaridad, situación que se relaciona al "efecto Mateo" del pasaje bíblico que describe el fenómeno del rico-se-hace-más-rico y el-pobre-se-hace-máspobre (Walberg \& Tsai, citado en Cunningham \& Stanovich, 2007). En este sentido, podemos coincidir con la investigación educacional que señala que "en ámbitos educativos se produciría un efecto de acumulación de las diferencias, fenómeno por el cual quienes ingresan con mayor conocimiento desarrollarán tasas de aprendizaje superiores a las de quienes ingresan con menores habilidades" (Diuk \& Ferroni, 2012, p. 210). En consecuencia, los estudiantes que no logran alcanzar un desempeño adecuado en las habilidades evaluadas en el inicio de la etapa escolar están destinados a fracasar en la adquisición de una lectura funcional, más aún si las familias de estos niños tienen dificultades económicas que impiden apoyar cognitivamente a sus hijos. Cabe destacar que, si bien la condición de vulnerabilidad social no fue estudiada en esta investigación, los niños y niñas que participan de ella pertenecen en su mayoría a escuelas municipales, que atienden a la población de los quintiles más bajos.

Por otra parte, la percepción de los profesores en torno al nivel lector alcanzado por los estudiantes es negativa, ya que tanto los hombres como las mujeres a los 8 años superan el $70 \%$ en las categorías no lector y lectura silábica. Ahora bien, si a esta edad los profesores perciben este nivel de aprendizaje, sería conveniente determinar en otras investigaciones las acciones de intervención específicas que realizan en torno al desarrollo de conciencia silábica y fonémica en los niños que presentan un diagnóstico de FIL y que cursan entre primer y tercer año de enseñanza básica, en el entendido que la adquisición de la lectura es más que un proceso de decodificación, es una herramienta para aprender, adquirir nuevos conocimientos de forma libre y autónoma, que representa la diversidad de intereses más allá del simple derecho de acceder a esta, se trata más bien de un legado insustituible para el desarrollo de la humanidad.

En la actualidad, existe un creciente reconocimiento de que las habilidades fonológicas pueden estar íntimamente relacionadas con el aprendizaje de la lectura en ortografías no alfabéticas, que a su vez juegan un papel importante en la predicción de variaciones en la habilidad de lectura de palabras. Por otro lado, podemos afirmar que el desarrollo exitoso de las habilidades de lectura de palabras depende de que el niño posea representaciones fonológicas estructuradas fonémicamente (Anthony, Williams, McDonald $\&$ Francis, 2007). En este sentido, el desarrollo de representaciones fonológicas estructuradas fonémicamente es una base crítica para aprender a leer con éxito en una escritura alfabética; en consecuencia, si no se consigue desarrollar tales representaciones fonológicas estructuradas fonémicamente, esta sería una de las principales causas de las dificultades para aprender a leer que experimentan los niños en edad escolar, particularmente para el caso de los niños con FIL (Melby-Lervåg, Lyster \& Hulme, 2012).

\section{Conclusiones}

La valoración de FIL se presenta aún como una encrucijada taxonómica a escalas internacional y nacional, debido a su delimitación conceptual y a la 
cuantificación de sus implicancias. Por ello, sigue siendo un tema poco estudiado por la diversidad de causas y perfiles funcionales que impiden una adecuada conceptualización.

De acuerdo con Salvador-Carulla et al. (2013), la FIL se conceptualiza como el límite que separa el funcionamiento intelectual "normal" de las dificultades intelectuales y de desarrollo. Describe un nivel de inteligencia por debajo del promedio, con resultados generales entre -1.01 y $-2.00 \mathrm{DE}$ (Jankowska et al., 2014). A pesar de sus limitaciones, son estudiantes que aprenden más lentamente que sus compañeros (Ruhela, 2014; Wieland \& Zitman, 2016).

El funcionamiento intelectual limítrofe afecta a una pequeña parte de la población infantil, que desencadena múltiples dificultades que agravan desde el desempeño académico, hasta la calidad de vida que alcanzan las personas con dicha condición. En consecuencia, determinar las necesidades de apoyo es sustancial para revertir parte de las posibles dificultades. En este sentido, desarrollar en estos infantes habilidades de conciencia fonológica que les permitan acceder al aprendizaje lector podría ser una herramienta crítica en el desarrollo de su etapa estudiantil y en su contexto social. Por lo anterior, las escuelas son fundamentales en la pesquisa de las posibles dificultades que se presentan en los inicios de la escolaridad, donde la FIL y las características de habilidades fonológicas que estos niños y niñas desarrollan son un punto determinante en la adquisición del código alfabético que les permita a estos estudiantes progresar curricular y socialmente en la escuela y la comunidad, respectivamente.

\section{Referencias}

Água-Dias, A. (2016). Características neuropsicológicas de alunos com funcionamento intelectual borderline. (Tesis de maestría, Universidade Coimbra, Brasil).
Alvarán, L., Sánchez, D., \& Restrepo-Ochoa, D. (2016). Neuropsicología de la inteligencia limítrofe. $\mathrm{Cua}$ dernos de Neuropsicología/Panamerican Journal of Neuropsychology, 10(2), 129-141.

Alegría, J. (2006). Por un enfoque lingüístico del aprendizaje de la lectura y sus dificultades. Infancia y Aprendizaje, 29(1), 93-111.

Anthony, J., Williams, J., McDonald, R., \& Francis, D. (2007). Phonological processing and emergent literacy in younger and older preschool children. Annals of Dyslexia, 57, 113-137. http:// doi.org/10.1007/s11881-007-0008-8

Aravena, J. (2014). Conciencia fonológica en dos grupos de escolares que cursan primer año básico en colegios municipales y particulares subvencionados. Revista Chilena de Fonoaudiología, 13, 40-49.

Atuesta, J., Vásquez, R., \& Urrego, Z. (2008). Aspectos psicopatológicos del coeficiente intelectual limítrofe: un estudio en el Hospital de la Misericordia, 2000-2005. Revista Colombiana de Psiquiatría, 37(2), 182-194.

Baglio, F., Cabino, A., Ricci, C., Baglio, G., Lipari, S., Griffanti, L., Preti, M., Nemni, R., Clerici, M., Zanette, M., \& Blasi, V. (2014). Abnormal development of sensory-motor, visual temporal and parahippocampal cortex in children with learning disabilities and borderline intellectual functioning. Frontiers in Human Neuroscience, 8(806), 1-11.

Bermúdez, R. (2010). Alteraciones en el neurodesarrollo causante de discapacidad cognitiva limítrofe y trastornos de aprendizaje. Revista Entérese Boletín Científico Universitario, (29), 20-24.

Bravo, L. (2016). El aprendizaje del lenguaje escrito y las ciencias de la lectura. Un límite entre la psicología cognitiva, las neurociencias y la educación. Límite. Revista Interdisciplinaria de Filosofia y Psicología, 11(36), 50-59.

Bravo-Valdivieso, L., Villalón, M., \& Orellana, E. (2004). Los procesos cognitivos en el aprendizaje de la lectura inicial: diferencias cognitivas 
entre buenos lectores y lectores deficientes. Estudios Pedagógicos, (30), 7-19.

Briones, G. (2002). Metodología de la investigación cuantitativa en las ciencias sociales. Instituto colombiano para el fomento de la educación superior. Bogotá: ICFES.

Cooter, K., \& Cooter, R. (2004). One size doesn't fit all: Slow learners in the reading classroom. The Reading Teacher, 57(7), 680-684.

Cruz, J., Almeida, M., Pinto, P., Constante, A., Macedo, A., Amaral, J., Monteiro, L., Lopes, E., y Ferreira, C. (2014). Contribuições da literacia emergente para o desempenho em leitura no final do $1^{\circ} \mathrm{CEB}$. Análise Psicológica, 32(3), 245-257.

Cunningham, A. y Stanovich, K. (2007). Los efectos de la lectura en la mente. Estudios Públicos, (108), 207-227.

Darling-Hammond, L. (2012). Educar con calidad y equidad: los dilemas del siglo XXI. Santiago: Fundación Chile.

Diuk, B., \& Ferroni, M. (2012). Dificultades de lectura en contextos de pobreza: ¿un caso de Efecto de Mateo. Revista Semestral da Associação Brasileira de Psicologia Escolar e Educacional, 16(2), 209-217.

Feld, V. (2014). Las habilidades fonológicas, su organización neurofisiológica y su aplicación en la educación. Pensamiento Psicológico, 12(1), 71-82.

Flores, R. Castro, J., \& Arias, N. (2011). Propiedades psicométricas de la prueba de procesamiento fonológico Profon en niños de 4 a 7 años de edad. Avances en Psicología Latinoamericana, 29(2), 240-257.

Flórez-Romero, R., Torrado-Pacheco, M. C., Arévalo-Rodríguez, I., Mesa-Guechá, C., Mondragón-Bohórquez, S., \& Pérez-Villegas, C. (2005). Habilidades metalingüísticas, operaciones metacognitivas y su relación con los niveles de competencia en lectura y escritura: un estudio exploratorio. Forma y función, (18), 15-44.
Hassiotis, A. (2015). Borderline intellectual functioning and neurodevelopmental disorders: prevalence, comorbidities and treatment approaches. Advances in Mental Health and Intellectual Disabilities, 9(5), 275-283.

Hernández, R. Fernández, C. Baptista, P. (2010). Metodología de la investigación. Mexico D. F.: McGraw Hill.

Jankowska, A. M., Bogdanowicz, M., \& Takagi, A. (2014). Stability of WISC-R scores in students with borderline intellectual functioning. Health Psychology Report, 2(1), 49-59. https://doi. org/10.5114/hpr.2014.42789

Karande, S., Kanchan, S., \& Kulkarni, M. (2008). Clinical and psychoeducational profile of children with bordeline intellectual functioning. Indian Journal of Pediatrics, 75, 795-800.

Van Kleeck, A. (1994). Metalinguistic development. En G. P. Wallach, \& K. G. Butler (Eds.), Language learning disabilities in school-age children and adolescents: Some principles and applications (pp. 53-98). Nueva York: Pearson.

Lazcano-Ponce, E., Katz, G., Allen-Leigh, B., Magaña Valladares, L., Rangel-Evode, G., Minoletti, A., Wahlberg, E., Vásquez, A., \& Salvador-Carulla, L. (2013). Trastornos del desarrollo intelectual en América Latina: un marco para establecer prioridades políticas de investigación y atención. Revista Latinoamericana de Salud Pública, 34(2), 204-209.

Malik, N., Rehman, G., \& Hanif, R. (2012). Effect of academic interventions on the developmental skills of slow learners. Pakistan Journal of Psychological Research, 27(1), 135-151.

Medina, A., \& Gajardo, A. (2010). Pruebas de comprensión lectora y producción de textos (CL-PT). Santiago, Chile: Ediciones UC.

Melby-Lervåg, M., Lyster, S., \& Hulme, C. (2012). Phonological skills and their role in learning to read: A meta-analytic review. Psychological Bulletin, 138(2), 322-352. http://dx.doi. org/10.1037/a0026744 
MINEDUC. (2015). Plan Nacional de Lectura 20152020. Recuperado de http://plandelectura.gob. cl/wp-content/uploads/2016/12/Plan-Nacional-Lectura-web-6-12-2016.pdf

Molina, G. (2016). Análisis teórico-reflexivo sobre los factores que intervienen en la calidad de los aprendizajes y práctica docente. Gestión de la educación, 6(1) 103-119.

Peltopuro, M., Ahonen, T., Kaartinen, J. Seppala, H., \& Narhi, V. (2014). Bordeline intellectual functioning: A systematic literature review. Intellectual and Developmental Disabilities, 52(6), 419-443.

Porta, M. (2012). Un programa de intervención pedagógica en conciencia fonológica. Revista de Orientación Educacional, 26(50), 93-111.

Porta, M., \& Ison, M. (2011). Hacia un enfoque comprensivo del aprendizaje lingüístico inicial como proceso cognitivo. Revista Iberoamericana de Educación, (55), 243-260.

Reis, A., Proença, M., \& Alves-Martins, M. (2015). Relação entre o nível conceptual de escrita, o conhecimento do nome das letras e a consciência silábica e fonêmica em crianças de idade pré-escolar. En L. Mata, M. Alves Martins, V. Monteiro, J. Morgado, F. Peixoto, A.C. Silva, \& J. Silva (Orgs.), Atas XIII Colóquio Internacional de Psicologia e Educação (pp. 284-298). Lisboa: ISPA.

Ruhela, R. (2014). The pain of slow learners. Online International Interdisciplinary Research Journal, 4(4), 193-200.
Salvador-Carulla, L., García-Gutiérrez, J. C., Gutiérrez-Colosía, M. R., Artigas-Pallarès, J., Ibáñez, J. C., Pérez, J. G., \& Martínez-Leal, R. (2013). Funcionamiento intelectual límite: Guía de consenso y buenas prácticas. Revista de Psiquiatría y Salud Mental, 6(3), 109-120. http://dx.doi. org/10.1016/j.rpsm.2012.12.001

Santos, M., \& Barrera, S. (2017). Impacto do treino em habilidades de consciência fonológica na escrita de pré-escolares. Psicología Escolar e Educacional, 21(1), 93-102.

Santos, S., \& Morato, P. (2012). ¡Acertando o passo! Falar de deficiência mental é um erro: Deve falar-se de dificuldade intelectual e desenvolvimental (DID). Por quê? Revista Brasileira de Educação Especial, 18(1), 3-16. Recuperado de http://www.scielo.br/pdf/rbee/v18n1/ a02v18n1.pdf

Valdivia, A. Medina, L., \& San Martín, E. (2014). Prácticas pedagógicas para la enseñanza de la lectura inicial: un estudio en el contexto de la evaluación docente chilena. PSYKHE, 23(2), 1-13.

Varela, V., \& De Barbieri, Z. (2015). Prueba de Evaluación de Conciencia Fonológica PECFO. Santiago de Chile: Ediciones UC.

Wieland, J., \& Zitman, F. G. (2016). It is time to bring borderline intellectual functioning back into the main fold of classification systems. BJPsych Bulletin, 40(4), 204-206. 